\title{
VISUAL-INERTIAL SENSOR FUSION TO ACCURACY INCREASE OF Autonomous Underwater Vehicles Positioning
}

\author{
Dmitrii Stepanov, Alexander Popov, Dmitrii Gromoshinskii \& Oleg Shmakov
}
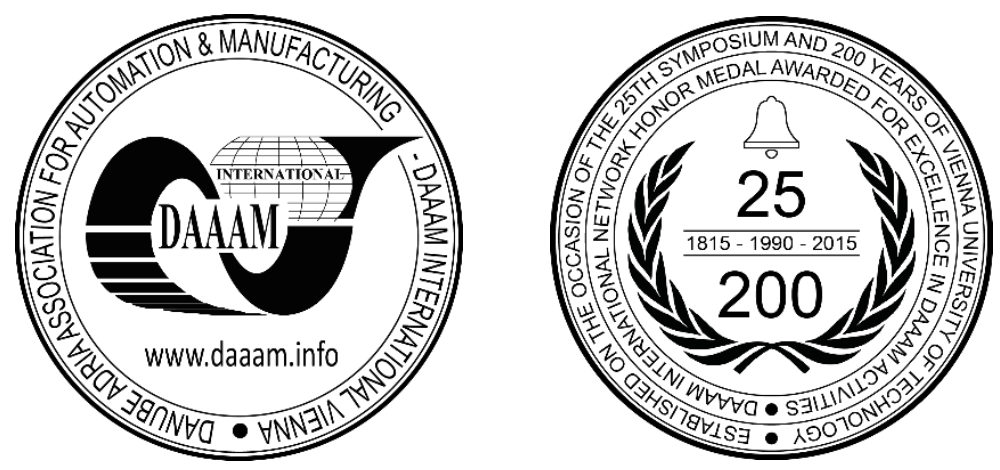

This Publication has to be referred as: Stepanov, D[mitrii]; Popov, A[lexander]; Gromoshinskii, D[mitrii] \& Shmakov, O[leg] (2018). Visual-Inertial Sensor Fusion to Accuracy Increase of Autonomous Underwater Vehicles Positioning, Proceedings of the 29th DAAAM International Symposium, pp.0615-0623, B. Katalinic (Ed.), Published by DAAAM International, ISBN 978-3-902734-20-4, ISSN 1726-9679, Vienna, Austria

DOI: $10.2507 / 29$ th.daaam.proceedings.089

\begin{abstract}
The article deals with the model of autonomous underwater vehicle (AUV) positioning relative to elements of underwater structures during inspection works at marine industrial facilities. The proposed approach is based on the principle of relative position determination using a set of active visual landmarks with a known spatial configuration relative to the structures and television cameras of the AUV. New methods of visual and inertial sensors fusion proposed to solve the problem in a case of incomplete landmarks visibility, as well as ways to improve the accuracy of the solution in the presence of partial information about the mutual position of the objects. The developed software for modeling of various landmarks systems and testing of the positioning algorithms, as well as the results of the experimental studies are described.
\end{abstract}

Keywords: autonomous underwater vehicle; computer vision; perspective-n-point; robot navigation; automatic docking

\section{Introduction}

Determining the position of underwater vehicles (AUVs) relative to objects of underwater infrastructure is an actual problem [1], since many tasks solved by the AUVs are directly related to the interaction with various objects. Examples of such tasks are manipulation operations, docking, inspection performed in automatic or automated mode.

At a close distance (up to 10-20 meters depending on water transparency) data from television cameras of the AUV can be used for the position determination. The problem of determining the position of an AUV using a television system is similar to other similar tasks and resembles, for example, the determination of the relative position of spacecrafts [2] or mobile robots (AGV). The principal difference between the system for determining the position of an AUV from [2] and from the AGV is that at depth or at night, due to low light and light propagation characteristics, it is difficult to use natural visual features of the observed objects. This leads to the need to use special light markers, clearly distinguishable by the television cameras underwater.

To solve the problem of determining of the position the light markers should be inside of the cameras field of view (FOV) and be clearly visible at the both the far border of the operating range of the system (about 10-20 meters, depending on the transparency of the water) and the near border (about 0.5 meters). 
When creating a marker system, it is necessary to consider the possible restrictions on the complexity and dimensions of such a marker system, as well as the observability of markers. In this regard, there is an actual situation when only a part of the parameters of the AUV position can be determined with sufficient accuracy using the marker system. Most of the AUVs are equipped with an IMU that provides a sufficiently accurate angular orientation of the AUV. By combining the data of the television system and the IMU, it is possible to solve the problem of finding the position of the AUV by just two markers observed by one television camera. In the presence of four or more distinguishable markers in the field of view of the camera, the problem can be solved without knowing the angles of roll and trim [3][ 4]. Thus, the task of the authors of the article is to create a system of artificial landmarks, and in addition, an approach allowing to determine the location of the underwater automatic vehicle, according to these landmarks, in conditions of obstructed visibility of the underwater space.

\section{Considering the markers positions}

A number of papers describe the use of flat targets with markers placed on a circle [16] or otherwise [13][14][15]. The main problem of such targets is the low accuracy of determining the angular orientation of the AUV relative to the object. An alternative solution designed to improve the accuracy of AUV orientation determination is the creation of targets forming a spatial structure [17]. While this solution provides the possibility of more accurate calculation of the angles of orientation of an AUV without the use of IMU, its implementation is not always possible due to a large size of a structure. In addition to light markers, the use of contrast passive targets of various shapes was studied. In the article [18] the variants of cruciform, circular and rectangular plane markers are considered.

Practical studies have shown that the visibility of the markers depends on a number of factors, first of which are the transparency of the water (loss of contrast of the image of the object with the distance), as well as the illumination of the marker. When using passive targets, it is necessary to provide their illumination from the AUV side, which is associated with a number of optical (light reflection from impurities on the way to the object with a corresponding decrease in contrast) and design problems. The use of active light targets can completely eliminate the problem of illumination of targets and achieve maximum contrast, depending only on the transparency of the water. At the same time, experimental studies have shown the achievable detection range of light markers in the range from 0.5 to 20 meters without their reconfiguration. Another factor to consider when designing a marker system is the ability to observe a sufficient number of key points (at least 2) over the entire operating range, considering possible AUV deviations, as the camera field of view increases linearly with the distance from a camera to the markers. For this, it is necessary to provide several groups of markers that will be used at different distances. The proposed solution involves the use of markers with the number of points not less than 4, the markers are grouped by 2, and for each of the two cameras a group of the markers is created. When working in the far zone, each camera observes all four markers (if the water transparency allows they are visible as 16 points, otherwise - as 4 light blobs). When working in the middle zone, each camera observes two markers. Finally, in the near zone there is one marker consisting of several points in the field of view of each camera.

Color or geometric coding can be used to simplify the task of distinguishing of the markers. As practical studies have shown, the perception of the color of markers is very dependent on the properties of the water, and therefore color coding cannot be considered as a reliable solution. As an alternative, the use of markers with different points positions is proposed.

\section{Automatic detection of markers and their grouping}

To ensure autonomous navigation of the AUV, images from its television cameras are entered into the on-board computer memory, where they are processed using special software that implements the algorithms described below. For use on remote-operated underwater vehicles (ROV), it is potentially possible to solve the problem of calculating the position on the coastal (ship) part of the complex, where the image is transmitted. At the same time, there are quite strict requirements for the quality of the transmitted images and minimizing delays in the transmission of frames.

Markers detection is processed using binarization with an adaptive threshold followed by edge detection with automatically adapted parameters [5]. If necessary, several light spots are grouped into one by the DBSCAN clustering method [6] and the center of each marker is found. After detection of the markers the numbering of the points is executed. If the approximate angle of the AUV heading (from the AUV navigation system) is known, the points are numbered using the knowledge of this angle. Otherwise, the numbering is performed sequentially according to the part of the frame in which they are located.

\section{A method for determining of the relative position of a set of points on a television frame}

If there are four or more points in an image (with their known spatial coordinates relative to the coordinate system of the object on which they are located), it is possible to solve the PnP problem [7]. There are both methods for solving the problem for a fixed number of points [4][10] and methods that allow solving the problem for an arbitrary number of points (more than four) with arbitrary geometry [3][11]. Practical studies have shown that it is advisable to use, for example, the method proposed in [12], because it showed high accuracy, sufficient speed and stability. 


\section{A method of determining of the relative position with two points and the known roll and trim angles}

In the case where it is impossible to ensure the observation of a sufficient number of points to solve the PnP-problem, as well as the need to accurately determine the angles of mutual orientation of the AUV and the object, it is advisable to use a different approach.

At known angles of roll and trim of the AUV and the object the angle of their mutual heading on the television frame can be calculated. This requires that one of the AUV television cameras see at least two points of the marker system with known spatial coordinates.

Then it is possible to calculate the rotation matrix between the coordinate systems. And knowing the orientation of the AUV, the same image with the two points can be used to determine all three relative displacements.

Some PnP methods are capable of finding the position of an object by three points [7,8]. However, there are ambiguities, and the choice of the right solution is associated with computational and algorithmic difficulties [9]. The proposed method finds a solution for two points at known roll and trim angles.

Finding of the rotation matrix between the object and the AUV coordinate systems.

Let us know the roll and trim angles of an AUV and an underwater object: $a_{x 1}, a_{x 2}, a_{y 1}, a_{y 2}$. Fig. 1 shows XY-planes of the AUV and the object and horizontal plane axes with a zero mutual. The lines P11 and P12 are parallel to the plane 1. And the lines P21 and P22 are parallel to the plane 2. At the same time all these lines are located in a vertical plane perpendicular to the horizontal plane.

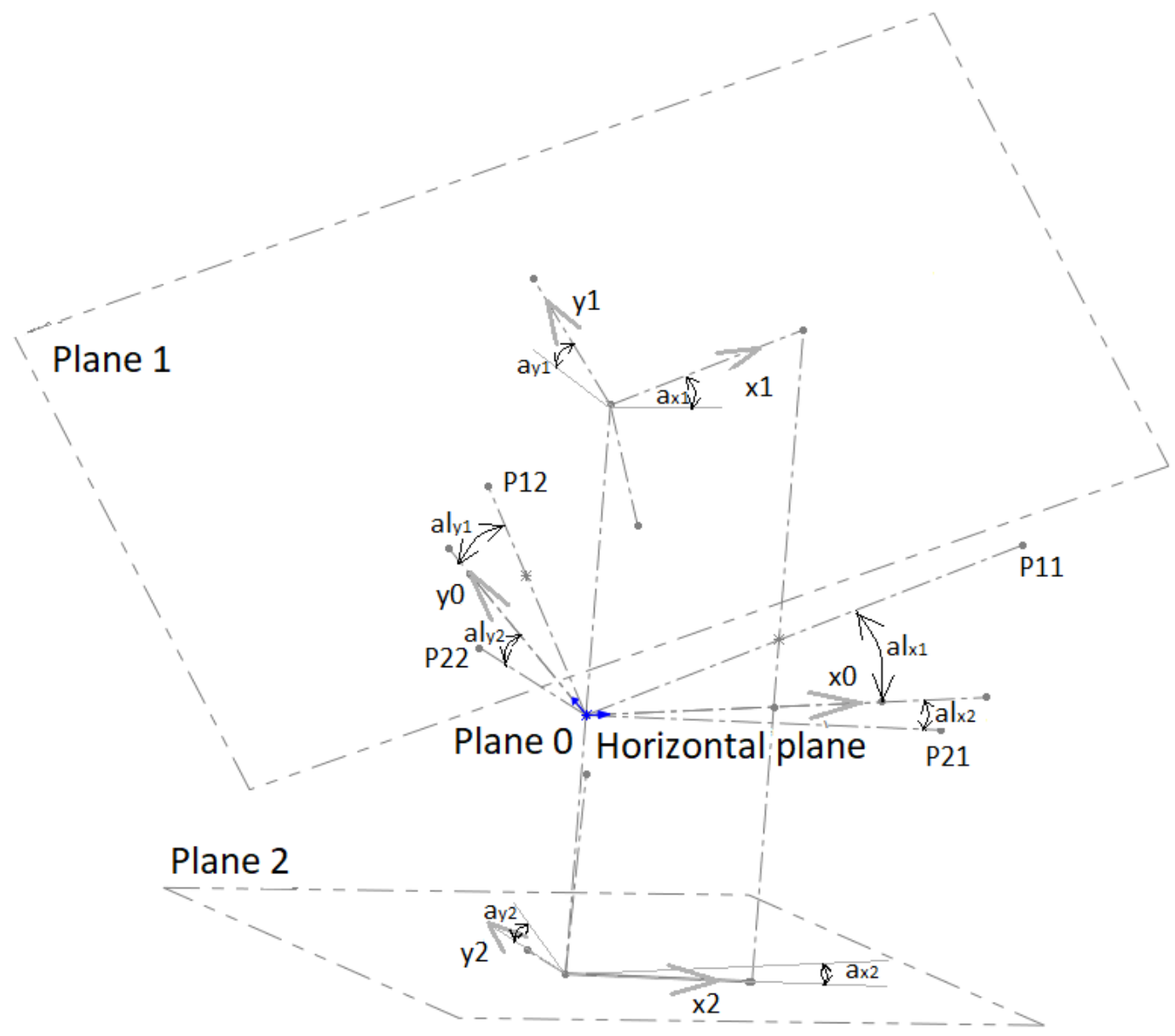

Fig. 1. Planes of the AUV and the object

Knowing the orts of the lines one can build the equations of the planes 1 and 2 relative to the horizontal plane. For now, we have taken the angle of the mutual heading equal to zero, just to demonstrate how to calculate the equations of the planes. One of these planes will simply be rotated to the desired angle after calculating of the angle of the mutual heading.

Let's calculate the auxiliary angles. $\mathrm{X}$ axes of the AUV and the object match the $\mathrm{x} 0$ plane of the horizon. So, the angles $a l_{x 1}, a l_{x 2}$ equals to the $a_{x 1}, a_{x 2}$. The angles $a l_{y 1}, a l_{y 2}$ are calculated as the fig. 2 shows: 


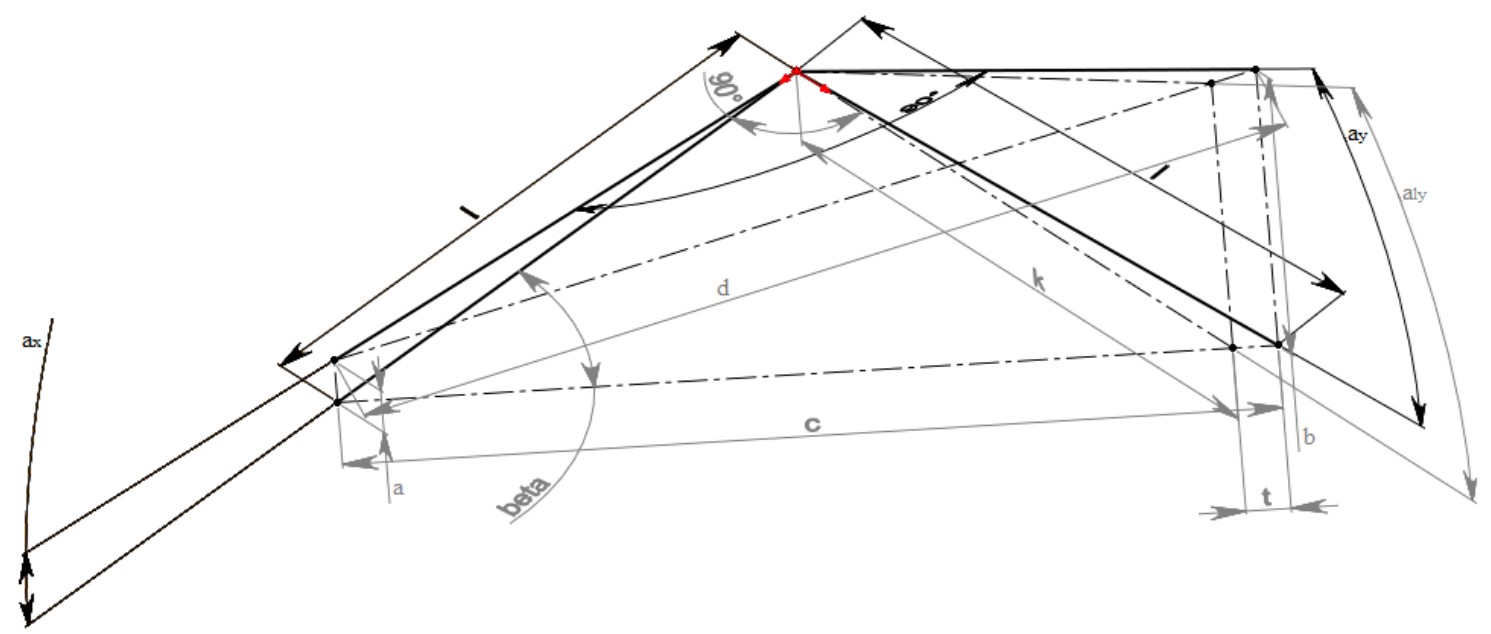

Fig. 2. Calculation of the ${ }^{a l_{y}}$ angle

From the trapezium $a b c d$ :

$$
\begin{aligned}
& c=l * \sqrt{\frac{1}{\cos \left(a_{x}\right)}+\frac{1}{\cos \left(a_{y}\right)}-\left(\operatorname{tg}\left(a_{x}\right)-\operatorname{tg}\left(a_{y}\right)\right)^{2}}, \\
& d=l * \sqrt{\frac{1}{\cos \left(a_{x}\right)}+\frac{1}{\cos \left(a_{y}\right)}} .
\end{aligned}
$$

Knowing $c$, one can calculate $\beta$ :

$$
\beta=\frac{\left(\pi-\arccos \left(\frac{\left.-(c)^{2}+2 * l^{2}\right)}{2 * l^{2}}\right)\right.}{2} .
$$

And considering $1-3$ the ${ }^{a l_{y}}$ angle is calculated as following:

$$
a l_{y}=\operatorname{arctg}\left(\operatorname{tg}\left(\alpha_{y}\right)+\left(c-l * \sqrt{\operatorname{tg}(\beta)^{2}+1}\right) * \operatorname{tg}\left(\arcsin \left(\frac{l *\left(\operatorname{tg}\left(\alpha_{x}\right)-\operatorname{tg}\left(\alpha_{y}\right)\right)}{d}\right)\right)\right)
$$

Calculation of the rotational angles $(5-11)$.

Knowing the $a l_{x 1}, a l_{x 2}, a l_{y 2}, a l_{y 2}$ one can write the planes 1 and 2 equations and find their normal coordinates:

$$
\begin{aligned}
& Z_{1}=n_{1}=\left(\begin{array}{c}
-\operatorname{tg}\left(a l_{x 1}\right) \\
-\operatorname{tg}\left(a l_{y 1}\right) \\
1
\end{array}\right) \\
& Z_{2}=n_{2}=\left(\begin{array}{c}
-\operatorname{tg}\left(a l_{x 2}\right) \\
-\operatorname{tg}\left(a l_{y 2}\right) \\
1
\end{array}\right)
\end{aligned}
$$

Let's also find the $\mathrm{x}$ axes of the planes: 


$$
\begin{aligned}
& X_{1}=\left(\begin{array}{c}
1 \\
0 \\
\operatorname{tg}\left(a l_{x 1}\right)
\end{array}\right) \\
& X_{2}=\left(\begin{array}{c}
1 \\
0 \\
\operatorname{tg}\left(a l_{x 2}\right)
\end{array}\right)
\end{aligned}
$$

And finally, there are the y axes:

$$
\begin{aligned}
& Y_{1}=Z_{1} * X_{1} \\
& Y_{2}=Z_{2} * X_{2}
\end{aligned}
$$

Let's normalize these vectors and build the rotation matrices:

$$
\begin{aligned}
& M_{01}=\left(\begin{array}{lll}
X_{1} & Y_{1} & Z_{1}
\end{array}\right) \\
& M_{02}=\left(\begin{array}{lll}
X_{2} & Y_{2} & Z_{2}
\end{array}\right)
\end{aligned}
$$

Now it's necessary to rotate one plane coordinate system (for example of the second plane) around the z0 axis of the horizontal plane. This can be done by applying of the following quaternion:

$$
\text { quat }=\left(\begin{array}{c}
0 \\
0 \\
1 \\
k_{-} \text {ang }
\end{array}\right)
$$

After the rotation we get the $M_{02 r}$ matrix. Now one can get the object to the AUV rotation matrix:

$$
M_{12}=M_{01} * M_{02 r}
$$

Knowing the rotation matrixes of the AUV relative to its camera coordinate system ${ }^{M_{k}}$ one can get the rotation matrix of the camera ralative tot he object:

$$
M_{k 2}=M_{k} * M_{12}
$$

Displacements calculation (12 - 18).

Knowing the rotation matrix and the image coordinates of the detected markers one can calculate the displacements and find the complete transition matrix. Let one of the poins to be $(0,0)$. If necessary this can be done by moving of the coordinate systems, doing all the calculations and moving the coordinate systems back. The fig. 3 describes the zx camera plane:

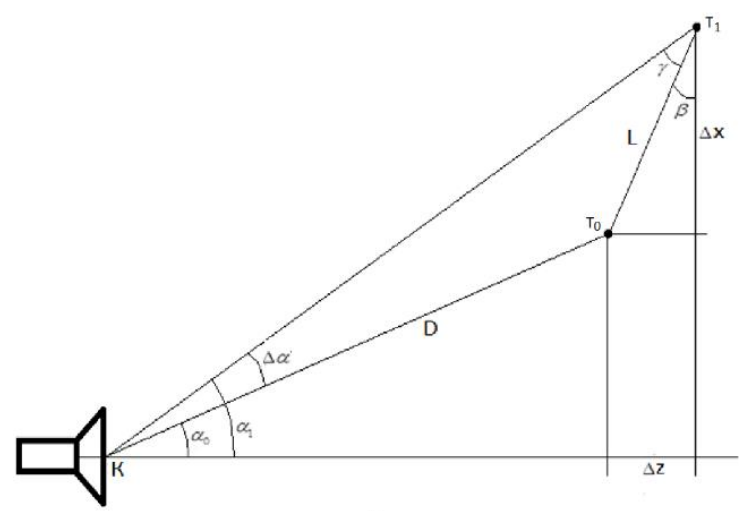

Fig. 3. Points in one of the camera planes 
Let us find the coordinates of the points relative to the camera coordinate system:

$$
\begin{aligned}
& T_{0}=M_{k 2} * T_{0}^{\prime} \\
& T_{1}=M_{k 2} * T_{1}^{\prime},
\end{aligned}
$$

where $T_{0}^{\prime}, T_{1}^{\prime}$ are the coordinates of the points relative to their own (markers) coordinate system. Knowing the internal camera parameters one can calculate the visible angles to these points:

$$
\begin{aligned}
& \alpha_{0 x}=\operatorname{arctg}\left(t_{0} \cdot x-x_{c} / f_{x}\right) \\
& \alpha_{1 x}=\operatorname{arctg}\left(t_{1} \cdot x-x_{c} / f_{x}\right)
\end{aligned}
$$

where ${ }^{t_{0}},{ }^{t_{1}}$ are the image points coordinates;

$x_{c}$ is the principal point $\mathrm{x}$ coordinate;

$f_{x}$ is the focal length.

Let's calculate the $L$ length:

$$
L_{x}=\sqrt{\left(T_{1} \cdot x-T_{0} \cdot x\right)^{2}+\left(T_{1} \cdot z-T_{0} \cdot z\right)^{2}}
$$

Angle $\beta$ :

$$
\beta_{x}=\operatorname{arctg}\left(\frac{T_{1} \cdot z-T_{0} \cdot z}{T_{1} \cdot x-T_{0} \cdot x}\right)
$$

Angle ${ }^{\gamma}$ :

$$
\gamma_{x}=\frac{\pi}{2}-\alpha_{1 x}-\beta_{x}
$$

Knowing the $\gamma$ angle one can calculate the $D$ line length using the cosine theorem:

$$
D_{x}=\frac{L_{x} * \sin \left(\gamma_{x}\right)}{\sin \left(\beta_{x}\right)}
$$

Knowing $D$ and the angles to the points one can calculate all the camera displacemets relative to the object coordinate:

$$
\begin{aligned}
& Z=\cos \left(\alpha_{0 x}\right) * D \\
& X=\sin \left(\alpha_{0 x}\right) * D \\
& Y=Z * \operatorname{tg}\left(\alpha_{0 y}\right)
\end{aligned}
$$

So, we have found the complete position and orientation of the AUV camera relative to the object's marker system.

\section{Finding the mutual heading angle:}

It remains to figure out how to initially determine the angle of the mutual heading $k_{-}$ang . This is done using the above model. Using one of the points one can make a guess about $\mathrm{X}$ and $\mathrm{Y}$ displacements of the camera relative to the object. Then the two points are reprojected to the image with a zero mutual heading and these displacements. The angle between the reprojected points and the x camera axis is calculated and compared to the same angle for the image points. Then the heading angle is fond by minimizing of the difference between the angles iteratively. 


\section{Correction of the angle of the mutual course according to data from several television cameras}

The angles of the roll and trim are taken with a high accuracy from the readings of the AUV's IMU, the angle of the mutual heading is determined directly from the testimony of the television cameras and is therefore dependent on the errors of finding of the markers on the frame. When the mutual position of the two objects using several cameras is obtained, it becomes possible to determine the angle of the mutual course independently. And for the method that uses two points for calculation, after finding the angle of the mutual course it becomes possible to refine the entire solution by restarting the method with the calculated angle.

Let us consider the case where there are two cameras. More cameras can always be reduced to this situation by taking them in pairs. After the calculation we have two transition matrices between the AUV and the object (markers) coordinate systems $M_{k 12}, M_{k 22}$.

Taking the coordinates of any of the points visible by the first camera $P_{1 t 2}$ and any of the points visible by the second camera $P_{2 t 2}$ one can get their coordinates relative to the cameras coordinate systems:

$$
\begin{aligned}
& P_{2 t 1 k}=M_{k 12} * P_{2 t 2} \\
& P_{1 t 2 k}=M_{k 22} * P_{1 t 2}
\end{aligned}
$$

The same coordinates can be obtained using the transition matrix from one camera to another:

$$
M_{k 1 k 2}=M_{k 1}^{-1} * M_{k 2}
$$

where $M_{k 1}, M_{k 2}$ are the transition matrices between the AUV's coordinate system and the cameras coordinate systems.

The resulting formula looks like:

$$
\begin{aligned}
& P_{12 k}^{\prime}=M_{k 1 k 2}^{-1} * M_{k 12} * P_{1 t 2} \\
& P_{21 k}^{\prime}=M_{k 1 k 2} * M_{k 22} * P_{2 t 2}
\end{aligned}
$$

So, we have two points calculate in two different ways: $\left(P_{1 t 2 k}{ }_{\text {и }} P_{12 k}^{\prime}\right)$ relative to the coordinate system of the second camera and $\left(P_{2 t 1 k}{ }_{\text {и }} P_{21 k}^{\prime}\right)$ relative to the coordinate system of the first camera. Let's find the distance between the points:

$$
\begin{aligned}
& P_{12 k}^{\prime}=M_{k 1 k 2}^{-1} * M_{k 12} * P_{1 t 2} \\
& P_{21 k}^{\prime}=M_{k 1 k 2} * M_{k 22} * P_{2 t 2}
\end{aligned}
$$

The least of the distances will show which camera results the more precise relative position calculation. Then the minimal $\mathrm{D}$ value is found for this camera by tuning of the mutual heading angle giving the optimal heading value.

\section{Computer model}

A computer model was developed to study the system and methods for determining the relative position of underwater objects using television cameras and light markers. The model is written in C++ using the Qt library. The computer model allows to set different configurations of markers, camera locations and parameters. Visually simulates a variety of cameras with markers and other objects in the scene. Allows to make various errors in the original data (pixel coordinates of points, in the angles of pitch and roll, etc.). It allows to test methods, both manually and automatically.

\section{Methods precision}

Precision, speed and robustness of the PnP-methods including the ones used in the described work are compared in details in [12]. Thus, here we just provide tables of RMS error dependency from the image coordinates error for the two methods used in this work. The method 1 is the proposed 2-points method with known pitch and roll angles. The method 2 is a PnP-method for 4 or more points. 


\begin{tabular}{|c|c|c|c|c|c|c|}
\hline $\begin{array}{c}\text { RMS error, } \\
\text { pix }\end{array}$ & $\mathbf{x , ~} \mathbf{m}$ & $\mathbf{y , ~} \mathbf{m}$ & $\mathbf{z ,} \mathbf{~ m}$ & $\mathbf{A x}^{\circ}$ & $\mathbf{A y} \boldsymbol{\circ}^{\circ}$ & $\mathbf{A z}{ }^{\circ}$ \\
\hline 0 & 0,000 & 0,000 & 0,000 & 0,000 & 0,000 & 0,002 \\
\hline 1 & 0,007 & 0,007 & 0,046 & 0,000 & 0,000 & 0,335 \\
\hline 2 & 0,015 & 0,013 & 0,091 & 0,000 & 0,000 & 0,463 \\
\hline 3 & 0,026 & 0,026 & 0,146 & 0,000 & 0,000 & 1,386 \\
\hline 4 & 0,048 & 0,033 & 0,235 & 0,000 & 0,000 & 1,456 \\
\hline 5 & 0,048 & 0,037 & 0,271 & 0,000 & 0,000 & 1,661 \\
\hline
\end{tabular}

Table 1. Method 1, 10m

\begin{tabular}{|c|c|c|c|c|c|c|}
\hline $\begin{array}{c}\text { RMS error, } \\
\mathbf{p i x}\end{array}$ & $\mathbf{x , ~} \mathbf{m}$ & $\mathbf{y , ~} \mathbf{m}$ & $\mathbf{z ,} \mathbf{~ m}$ & $\mathbf{A x} \boldsymbol{\circ}^{\circ}$ & $\mathbf{A y},^{\circ}$ & $\mathbf{A z}{ }^{\circ}$ \\
\hline 0 & 0,000 & 0,000 & 0,000 & 0,000 & 0,000 & 0,002 \\
\hline 1 & 0,001 & 0,002 & 0,006 & 0,000 & 0,000 & 0,090 \\
\hline 2 & 0,001 & 0,004 & 0,008 & 0,000 & 0,000 & 0,209 \\
\hline 3 & 0,004 & 0,006 & 0,017 & 0,000 & 0,000 & 0,203 \\
\hline 4 & 0,005 & 0,007 & 0,018 & 0,000 & 0,000 & 0,410 \\
\hline 5 & 0,006 & 0,011 & 0,030 & 0,000 & 0,000 & 0,544 \\
\hline
\end{tabular}

Table 2. Method 1,3m

\begin{tabular}{|c|c|c|c|c|c|c|}
\hline $\begin{array}{c}\text { RMS error, } \\
\text { pix } \\
\end{array}$ & $\mathbf{x}, \mathbf{m}$ & $\mathbf{y}, \mathbf{m}$ & $\mathbf{z}, \mathbf{m}$ & $\mathbf{A x},^{\circ}$ & $\mathbf{A y},^{\circ}$ & $\mathbf{A z},^{\circ}$ \\
\hline 0 & 0,000 & 0,000 & 0,000 & 0,000 & 0,000 & 0,000 \\
\hline 1 & 0,019 & 0,018 & 0,136 & 1,371 & 1,343 & 1,003 \\
\hline 2 & 0,036 & 0,029 & 0,259 & 2,533 & 2,758 & 1,808 \\
\hline 3 & 0,054 & 0,052 & 0,386 & 4,797 & 3,923 & 3,309 \\
\hline 4 & 0,072 & 0,053 & 0,464 & 5,353 & 5,190 & 3,905 \\
\hline 5 & 0,089 & 0,084 & 0,601 & 6,633 & 6,720 & 4,816 \\
\hline
\end{tabular}

Table 3. Method 2, 10m

\begin{tabular}{|c|c|c|c|c|c|c|}
\hline $\begin{array}{c}\mathbf{R} M S \text { error, } \\
\mathbf{p i x}\end{array}$ & $\mathbf{x , ~} \mathbf{m}$ & $\mathbf{y , ~} \mathbf{m}$ & $\mathbf{z , ~} \mathbf{m}$ & $\mathbf{A x} \boldsymbol{,}^{\circ}$ & $\mathbf{A y},^{\circ}$ & $\mathbf{A z} \mathbf{\circ}^{\circ}$ \\
\hline 0 & 0,000 & 0,000 & 0,000 & 0,000 & 0,000 & 0,000 \\
\hline 1 & 0,001 & 0,001 & 0,006 & 0,398 & 0,383 & 0,287 \\
\hline 2 & 0,001 & 0,001 & 0,013 & 0,926 & 0,734 & 0,629 \\
\hline 3 & 0,002 & 0,002 & 0,021 & 1,330 & 1,114 & 0,954 \\
\hline 4 & 0,003 & 0,003 & 0,025 & 1,661 & 1,354 & 1,129 \\
\hline 5 & 0,004 & 0,003 & 0,032 & 1,754 & 1,679 & 1,178 \\
\hline
\end{tabular}

Table 4. Method 2, 3m

The experiments show that the method 1 is more precise than the method 2 .

\section{Conclusion}

The presented approach based on the use of television cameras of an autonomous underwater vehicle together with an active marker system allows to determine the position of the AUV relative to an underwater object with high accuracy. The approach is effectively applied at distances for which special light marks are visible in the pictures from the television cameras. These distances obviously exceed the range of applicability of passive markers presented in other works due to light propagation in marine water. The approach implemented in the form of an AUV vision system was successfully tested using a computer model and experiments in a technical pool and in the open sea. 
The proposed method for determining of the relative position with two points on an image and two known angles of an AUV showed better results in accuracy than traditional PnP-methods. Due to the need to find just two points, it can be used in various systems to find a position of a robot (vehicle etc) relative to the scene with a small number of visible features with known geometry. The proposed method of refinement of the angle of the mutual heading according to data from several television cameras showed good results in improving the accuracy. The computer model developed for the study of the system allows to form images of different types of marker systems of active and passive type for different mutual positions of AUV and objects. The model can then be used to develop new methods for determining the position.

Further research directions are the following tasks: study the effect of changes in the configuration of markers on the accuracy of positioning, in addition, the use of the obtained methods for determining the relative position of flying drones (UAVs) during take-off, landing or maneuvering in the immediate vicinity of the object.

\section{Acknowledgments}

The results were obtained in the framework of a state task of the Ministry of Education and Science of the Russian Federation. Project 8.13276.2018/12.1

\section{References}

[1] Bakhshiev, A.V. and others.(2010) Proposals development for the design and technical implementation of the determining parameters relative motion system based by video processing. - Technical report. RTC. - SaintPetersburg, 2010. - pp: 143.

[2] Stepanov, D., Bakhshiev, A., Gromoshinskii, D., Kirpan, N., \& Gundelakh, F. (2015). Determination of the relative position of space vehicles by detection and tracking of natural visual features with the existing TV-cameras, International Conference on Analysis of Images, Social Networks and Textspp, 431-442, Springer, Cham

[3] Moreno-Noguer, F., Lepetit, V., \& Fua, P. (2007). Accurate non-iterative o (n) solution to the pnp problem. In Computer vision, 2007. ICCV 2007. IEEE 11th international conference, pp. 1-8,. IEEE, Rio de Janeiro.

[4] Horaud, R., Conio, B., Leboulleux, O., \& Lacolle, B. (1989). An analytic solution for the perspective 4-point problem. In Computer Vision and Pattern Recognition, 1989. Proceedings CVPR'89, IEEE Computer Society Conference, pp. 500-507. IEEE

[5] Suzuki, S. (1985). Topological structural analysis of digitized binary images by border following, Computer vision, graphics, and image processing, 30(1), pp. 32-46

[6] Ester, M., Kriegel, H. P., Sander, J., \& Xu, X. (1996). A density-based algorithm for discovering clusters in large spatial databases with noise. Kdd, Vol. 96, No. 34, pp. 226-231

[7] Fischler, M. A., \& Bolles, R. C. (1981). Random sample consensus: a paradigm for model fitting with applications to image analysis and automated cartography. Communications of the ACM, 24(6), pp. 381-395

[8] Gao, X. S., Hou, X. R., Tang, J., \& Cheng, H. F. (2003). Complete solution classification for the perspective-threepoint problem. IEEE transactions on pattern analysis and machine intelligence, 25(8), pp. 930-943.

[9] Filaretov, V. F., Zhirabok, A. N., \& Zuev, A. (2011). The synthesis of robust feedback for diagnostic observers of navigation sensors of autonomus underwater vehicle, Annals of DAAAM \& Proceedings. pp. 3-4.

[10] Bujnak, M., Kukelova, Z., \& Pajdla, T. (2010). New efficient solution to the absolute pose problem for camera with unknown focal length and radial distortion, Asian Conference on Computer Vision, pp. 11-24, Springer, Berlin, Heidelberg.

[11] Hesch, J. A., \& Roumeliotis, S. I. (2011). A direct least-squares (DLS) method for PnP, Computer Vision (ICCV), 2011 IEEE International Conference, pp. 383-390. IEEE

[12] Kirpan, N.A. (2013) An algorithm of determination of position and orientation of an object with a reference target by a flat image registered at a space docking: MSc thesis. - Saint-Petersburg, 2013. - pp. 165.

[13] Palmer, Tomeu \& Romagós, David \& Ridao, Pere \& Mallios, Angelos. (2009). Vision based localization system for AUV docking on subsea intervention panels. 1 - 10. 10.1109/OCEANSE.2009.5278216.

[14] Krupinski, Szymon \& Maurelli, Francesco \& Mallios, Angelos \& Sotiropoulos, Panagiotis \& Palmer, Tomeu. (2009). Towards AUV docking on sub-sea structures. 1 - 10. 10.1109/OCEANSE.2009.5278144.

[15] Sotiropoulos, Panagiotis \& Grosset, D \& Giannopoulos, Georgios \& Casadei, F. (2009). AUV docking system for existing underwater control panel. OCEANS '09 IEEE Bremen: Balancing Technology with Future Needs. 1 - 5. 10.1109/OCEANSE.2009.5278201

[16] Park, J. Y., Jun, B. H., Kim, K., Lee, P. M., Oh, J. H., \& Lim, Y. K. (2009). Improvement of vision guided underwater docking for small AUV ISiMI, OCEANS 2009, MTS/IEEE Biloxi-Marine Technology for Our Future: Global and Local Challenges, pp. 1-5, IEEE.

[17] Yu, S. C., Ura, T., Fujii, T., \& Kondo, H. (2001). Navigation of autonomous underwater vehicles based on artificial underwater landmarks. In OCEANS, 2001. MTS/IEEE Conference and Exhibition, Vol. 1, pp. 409-416, IEEE.

[18] Boreyko A.A., Vorontsov A.V., Kushnerik A.A., Scherbatyuk A.F. (2010) Video processing algorithms for solving some problems of control and navigation of autonomous unmanned underwater vehicles, Underwater Research and Robotics. Vol. 1. pp. 29-39. 